\title{
Design of an SFQ Microwave Chopper for Controlling Quantum Bits
}

\author{
Go Matsuda, Yuki Yamanashi, and Nobuyuki Yoshikawa
}

\begin{abstract}
A microwave chopper using single-flux-quantum (SFQ) circuits is proposed for the control of quantum bits (qubits). The proposed microwave chopper is composed of a DC/SFQ converter, an SFQ switch and a band-pass filter (BPF). In operation, an externally applied microwave is input to a DC/SFQ converter to generate an SFQ pulse train, which is chopped at high speed by the SFQ switch. The SFQ pulse train is then filtered by the BPF to remove higher harmonics. The transient response, the amplitude and the spectrum of the microwave output from the chopper were examined by circuit simulation, taking into account the thermal noise. The chopper was implemented using the SRL $2.5 \mathrm{kA} / \mathrm{cm}^{2}$ $\mathrm{Nb}$ standard process and its characteristics were investigated. It was shown that the microwave output could be successfully chopped by the SFQ switch. The amplitude and line width of the microwave output were measured as $15 \mu \mathrm{V}$ and $2 \mathrm{~Hz}$ at $7 \mathrm{GHz}$.
\end{abstract}

Index Terms-Microwave switches, quantum bit, SFQ circuit, superconducting devices, superconducting integrated circuits.

\section{INTRODUCTION}

O UPERCONDUCTING quantum bits (qubits) are promising devices for the realization of a quantum computer, because they can be largely integrated using conventional fabrication processes [1]-[3]. Coherent control of superconducting qubits is generally achieved by applying coherent microwave pulses. For example, full rotation of the qubit state is obtained by application of a coherent $\pi$ pulse. The microwave pulse is required to be of high quality, i.e. narrow line width and low noise temperature, and to be switched with a high time resolution, better than tens of ps. In order to realize such a microwave pulse, we have been developing a scheme to chop an externally applied continuous microwave using single-flux-quantum (SFQ) circuits [4]. In this paper we will propose an SFQ microwave chopper for the purpose of qubit manipulation. The proposed SFQ microwave chopper has the following advantages:

i) High quality microwaves with narrow line width are obtained, because a reference microwave can be provided from a high-quality microwave oscillator at room temperature.

ii) The control of microwaves with high time resolution is possible due to the high-speed operation of the SFQ circuits.

Manuscript received August 29, 2006. This work was supported by CREST, Japan Science and Technology Agency.

The authors are with the Department of Electrical and Computer Engineering, Yokohama National University, Yokohama 240-8501, Japan (e-mail: nyoshi@ynu.ac.jp).

Color versions of one or more of the figures in this paper are available online at http://ieeexplore.ieee.org.

Digital Object Identifier 10.1109/TASC.2007.898657

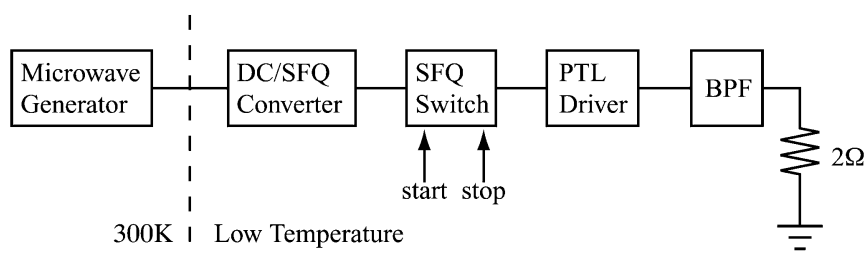

Fig. 1. Block diagram of an SFQ microwave chopper.

iii) The microwave chopper can be integrated with qubits at a similar temperature stage.

All these features enable the realization of a large qubit system, where each qubit can be precisely and individually controlled by coherent microwaves.

In our qubit system, we plane to mount the chopper in the separate microwave module, which is placed on the same temperature stage of the dilution refrigerator as the qubit module is placed on. The microwave from the chopper module is provided to the qubit module through coaxial cable, and applied to the qubit by transmission line.

\section{SFQ MicROWAVE CHOPPER}

Fig. 1 shows a block diagram of the proposed SFQ microwave chopper. The chopper is composed of a microwave generator at room temperature, a DC/SFQ converter, an SFQ NDRO-based switch, a passive transmission line (PTL) driver [5], [6], and a band-pass filter (BPF). In operation, an externally applied microwave is input to the DC/SFQ converter, which generates an SFQ pulse at every raised edge of the microwave. The SFQ pulse train generated by the DC/SFQ converter is turned on and off by the SFQ switch, which is controlled by application of start or stop SFQ inputs. The output SFQ pulse train is then passed to the PTL and filtered by the BPF to remove higher harmonics. The resultant microwave has the same frequency as the externally applied microwave, and is chopped by the SFQ switches.

We have examined the operation of the SFQ chopper by circuit simulations. The WRspice circuit simulator [7] is used in this study. Fig. 2 shows simulated output waveforms at each component of the chopper. In the simulation, a $10 \mathrm{GHz}$ microwave is applied to the chopper. The impedance of the PTL and BPF is assumed to be $2 \Omega$. The BPF is made by cascading a low-pass filter (LPF) and a high-pass filter (HPF), where the LPF is a 9th-order Chebyshev filter with a $13 \mathrm{GHz}$ cutoff frequency, and the HPF is a 3rd-order K-type filter with a $5 \mathrm{GHz}$ cutoff frequency. It can be seen from Fig. 2 that periodical SFQ pulses are generated from the DC/SFQ converter, which are turned on and off by the SFQ switch by applying start/stop 


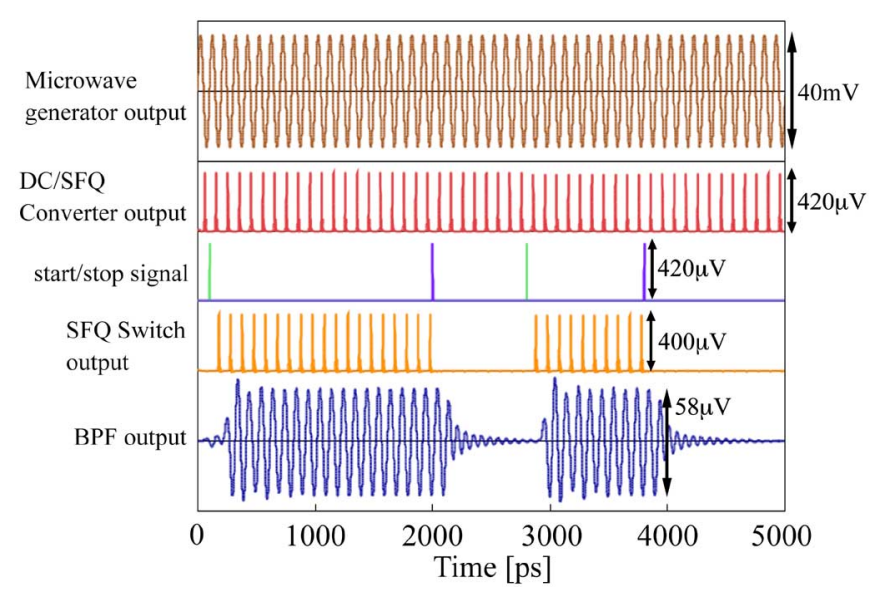

Fig. 2. Simulation result of an SFQ microwave chopper.

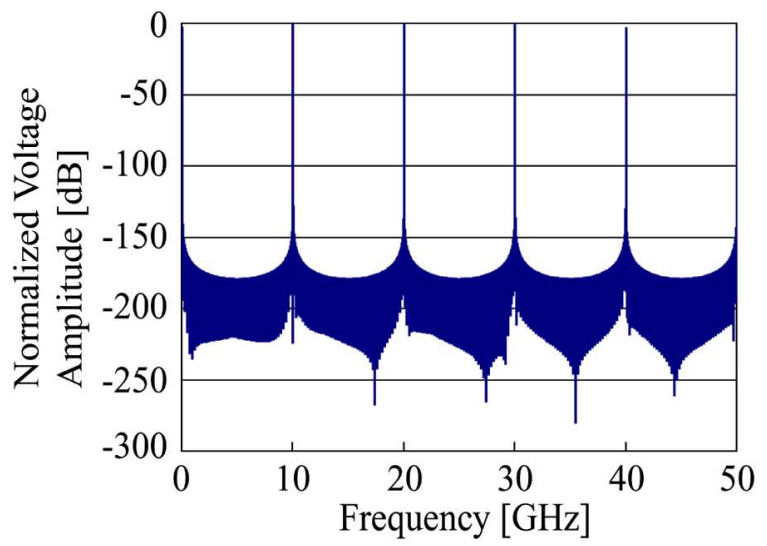

(a)

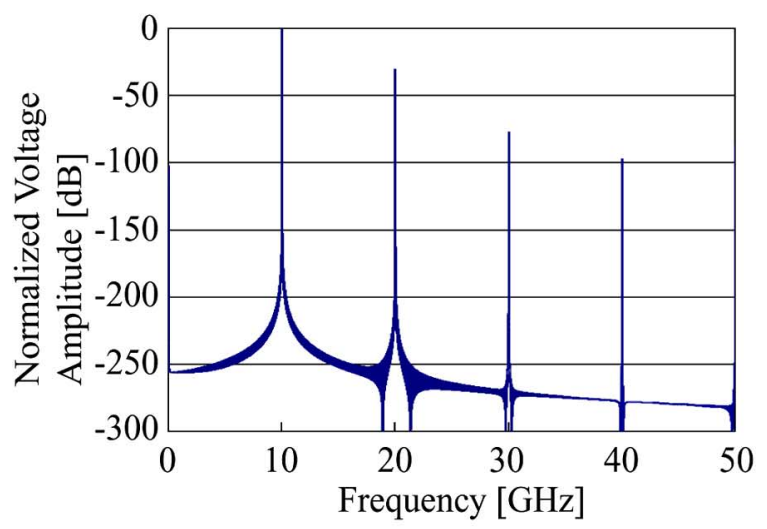

(b)

Fig. 3. (a) The spectrum of the output signal from the DC/SFQ converter. The reference voltage level is $34 \mu \mathrm{V}$. (b) The spectrum of the output signal from the BPF. The reference voltage level is $29 \mu \mathrm{V}$.

signals. The SFQ pulse trains are converted to sinusoidal microwave pulses by the BPF. The voltage amplitude and the rise and fall time of the microwave pulse are evaluated from the simulation to be $29 \mu \mathrm{V}$ and $90 \mathrm{ps}$.

\section{SPECTRUM ANALYSIS}

We have calculated the spectrum of the microwave output from the chopper to examine its quality. Fig. 3(a) and (b) show the spectrum of the SFQ pulse train from the DC/SFQ converter, and the microwave output from the BPF, respectively. In

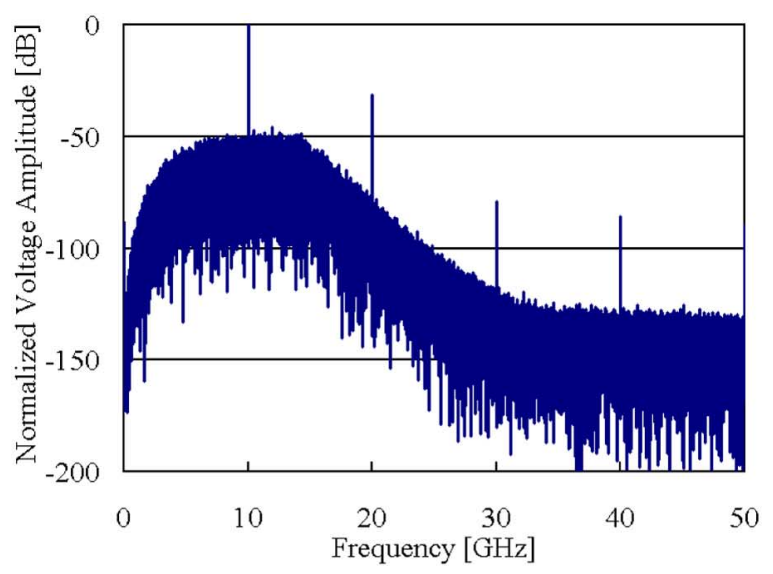

(a)

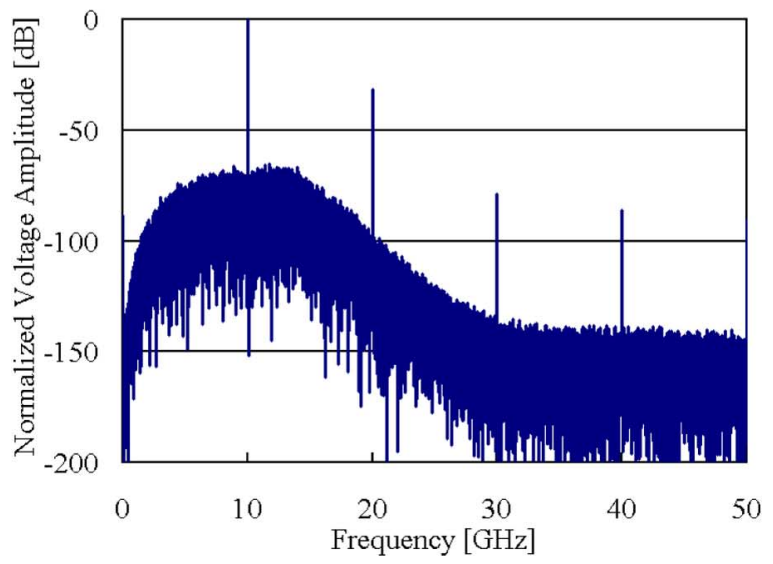

(b)

Fig. 4. The spectrum of the output signal from the BPF taking into account the thermal noise at every resistor. (a) $T=4 \mathrm{~K}$. The reference voltage level is $29 \mu \mathrm{V}$. (b) $T=50 \mathrm{mK}$. The reference voltage level is $29 \mu \mathrm{V}$.

Fig. 3(a) a sharp peak appears at $10 \mathrm{GHz}$ in addition to its higher harmonics, with amplitudes of almost the same value, while the higher harmonics are significantly reduced in Fig. 3(b). For example, the amplitude of the second harmonic at $20 \mathrm{GHz}$ was reduced by $30 \mathrm{~dB}$.

We have also investigated the effect of thermal noise or Johnson noise on the quality of the microwave output. In the simulation, stochastic random current noise was added to every resistor in the circuit using WRspice. The rms value of the current noise is given by

$$
I_{N}=\sqrt{4 k_{B} T B / R}
$$

where $T$ is the operating temperature, $R$ is the resistance, and $B$ is the bandwidth of the system [8]. Fig. 4 shows the spectra of the microwave output from the BPF at different temperatures, taking into account the thermal noise. One can see that the noise floor level gets smaller with a decrease in the temperature. It was confirmed from the temperature dependence of the spectrum that the noise-voltage density, at approximately $10 \mathrm{GHz}$ in Fig. 4, is given by $\left(4 k_{\mathrm{B}} T R\right)^{1 / 2}$, where $R$ is the shunt resistance of the PTL driver. It can be also seen from the simulation results that the line width of the spectrum is not affected by the thermal noise. This is because the random phase fluctuation of the output microwave caused by the jitter of the Josephson junction is averaged and 


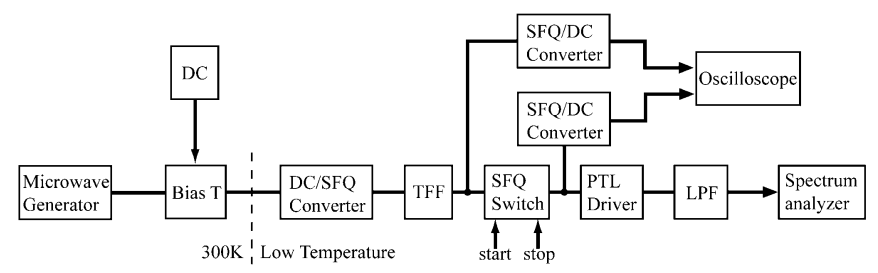

Fig. 5. Block diagram of the measurement system.

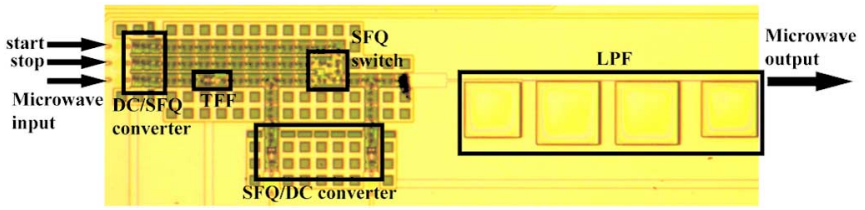

Fig. 6. Photograph of the SFQ microwave chopper.

canceled out in the long-term spectrum measurement. It should be noted that the short-term phase fluctuation caused by the jitter still exists and may affect the coherent state of the qubit.

\section{EXPERIMENT}

Fig. 5 shows a block diagram of the measurement system for the SFQ microwave chopper. In the test system, the microwave frequency was reduced to half of the input microwave frequency by inserting a T flip-flop (T-FF) between the DC/SFQ converter and the SFQ switch in order to eliminate the crosstalk between the input and output microwave lines. To confirm operation of the SFQ circuit, output signals from the T-FF and the SFQ switch were monitored using SFQ/DC converters. The microwave output from the chopper is directly measured by a spectrum analyzer. A bias- $\mathrm{T}$ at the input is required to add the offset voltage to the input microwaves for the operation of the DC/SFQ converter. The BPF of the chopper is replaced with a LPF in the test circuit to check the functionality of the SFQ circuit at low frequency. The LPF is a 9th-order Chebyshev filter with a $13 \mathrm{GHz}$ cutoff frequency. The impedance of the superconducting microstripline and the LPF were designed to be $2 \Omega$ and are connected to $50 \Omega$ microwave lines through the on-chip impedance matching circuit. The chip is mounted on a BCP-2 cryoprobe, a wide-bandwidth probe manufactured by American Cryoprobe Inc. The signal attenuation in the probe was estimated to be approximately $-3.5 \mathrm{~dB}$ at $10 \mathrm{GHz}$. The SFQ microwave chopper was implemented using the SRL $2.5 \mathrm{kA} / \mathrm{cm}^{2} \mathrm{Nb}$ standard process [9]. Fig. 6 shows a photograph of the chopper. The total junction number is 121 and the circuit area is $417 \times 1740 \mu \mathrm{m}^{2}$.

We first measured the functionality of the SFQ circuit at low speed. Fig. 7 shows the output signal from the SFQ switch when the frequency of the input rf wave is $50 \mathrm{MHz}$. Because each transition of the output signals corresponds to the output of the SFQ pulses, the frequency of the output SFQ train is $25 \mathrm{MHz}$, which is half of the input rf wave frequency. We also confirmed that the output SFQ pulses from the SFQ switch were properly controlled by application of start and stop signals.

The spectrum of the microwave output from the chopper is shown in Fig. 8, where a $14 \mathrm{GHz}$ microwave was applied to the chopper. Fig. 8(a) and (b) show the microwave spectra when the SFQ switch is tuned on and off, respectively. A sharp peak

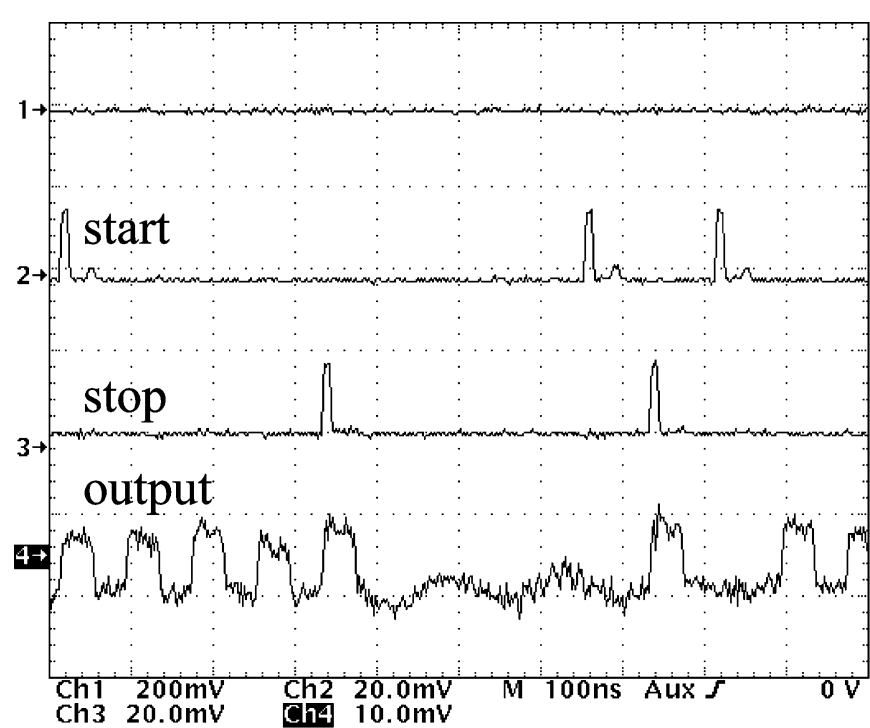

Fig. 7. The output signal from the SFQ switch when the frequency of the input rf wave is $50 \mathrm{MHz}$. Each rising edge in the "start" and "stop" signals correspond to the incidence of an SFQ pulse, and each transition in the "output" corresponds to the output of an SFQ pulse.

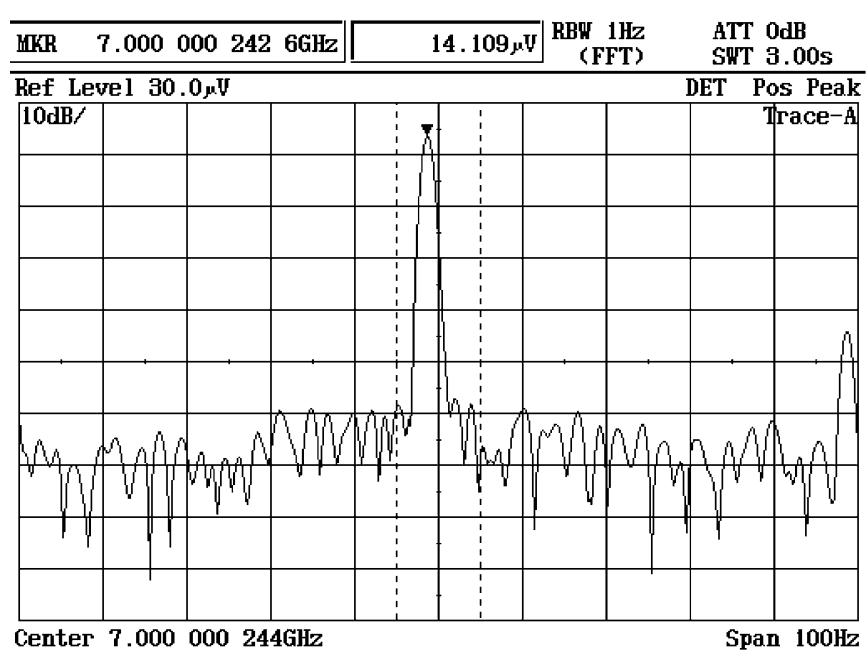

(a)

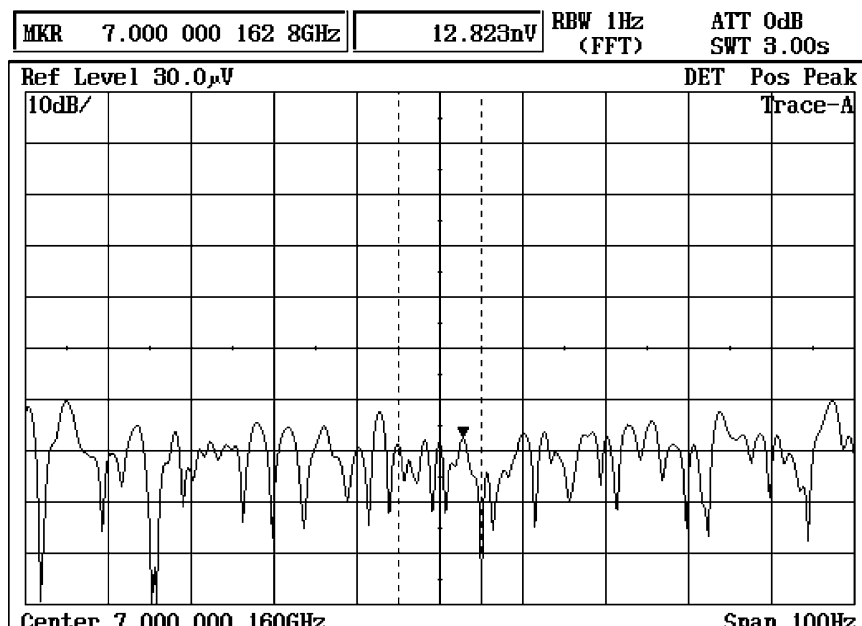

(b)

Fig. 8. The measured spectrum of the output microwave from the SFQ chopper when the SFQ switch is turned (a) on and (b) off. The output microwave frequency is $7 \mathrm{GHz}$. 


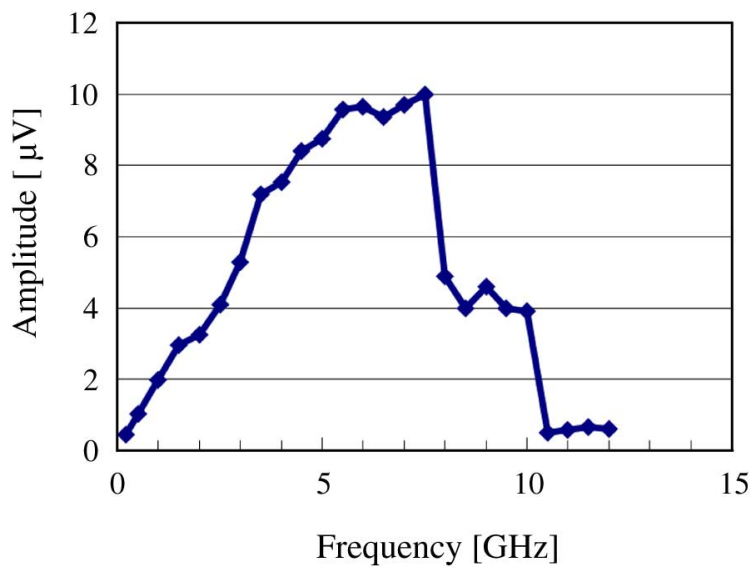

Fig. 9. The dependence of the voltage amplitude of the output microwave from the SFQ chopper on the microwave frequency. The bias current for the SFQ circuit is $17 \mathrm{~mA}$.

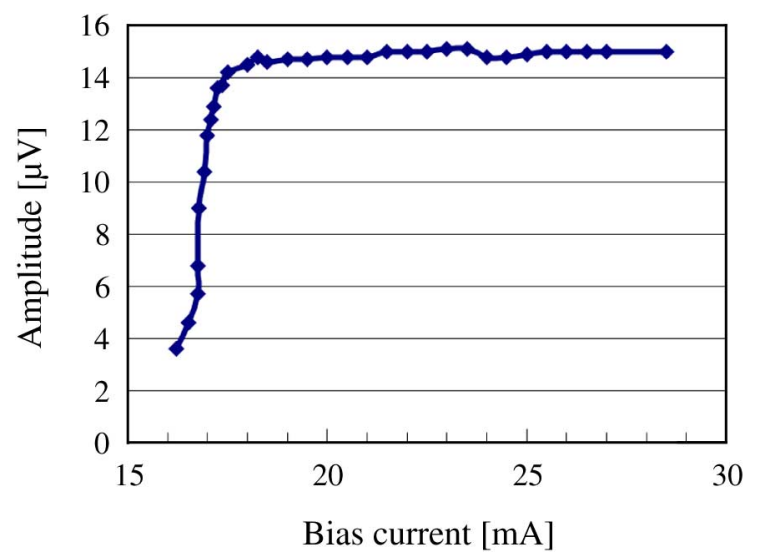

Fig. 10. The dependence of the voltage amplitude of the output microwave from the SFQ chopper on the bias current. The input microwave frequency is $14 \mathrm{GHz}$.

is observed at $7 \mathrm{GHz}$ when the SFQ switch is turned on. Note that the observed frequency is exactly equal to half of the input microwave frequency. The amplitude of the output microwave is $15 \mu \mathrm{V}$. This value is reasonable considering that the simulated microwave amplitude is $29 \mu \mathrm{V}$, and the signal attenuation in the probe is approximately $-3.5 \mathrm{~dB}$. The line width obtained from the spectrum is $2 \mathrm{~Hz}$, which is equal to that of the externally applied microwave.

Fig. 9 shows the dependence of the voltage amplitude of the output microwave on the microwave frequency. The microwave amplitude decreases with an increase in the frequency due to the LPF characteristics, so that the actual cut off frequency obtained from the test results seems slightly lower than the designed value, i.e. $13 \mathrm{GHz}$. At low frequency, the amplitude is proportional to the frequency. This is because the amplitude of the output microwave is proportional to the density of the SFQ pulse at low frequency.

The dependence of the voltage amplitude of the output microwave on the bias current of the SFQ circuits is shown in Fig. 10. One can see that the output voltage amplitude is almost constant and approximately $15 \mu \mathrm{V}$, except at the lower end of the bias current. The amplitude of the chopper is not changed sufficiently, so that use of a Josephson attenuator is planned, which will utilize the Josephson inductance as an rf circuit element in order to control the amplitude of the output microwave.

Heating up of the SFQ circuit at very low temperature is a crucial problem. Allowable power dissipation at $\mathrm{mK}$ temperature range was investigated in [10]. The power consumption of the SFQ microwave chopper, without the monitoring circuit, is estimated to be $9.6 \mu \mathrm{W}$. This can be reduced to approximately $0.1 \mu \mathrm{W}$ by decreasing the critical current of the Josephson junction and using the low-power technique [11].

\section{CONCLUSION}

We have proposed an SFQ microwave chopper to control superconducting qubits. We have confirmed the high-speed controllability of the microwave with a $90 \mathrm{ps}$ rise and fall time in the simulation. The spectrum analysis has shown that the line width of the output microwave is not influenced by the thermal noise. We have implemented the chopper using the $2.5 \mathrm{kA} / \mathrm{cm}^{2}$ $\mathrm{Nb}$ process and demonstrated correct operation. The amplitude and the line width of the output microwave were measured as $15 \mu \mathrm{V}$ and $2 \mathrm{~Hz}$ at $7 \mathrm{GHz}$.

\section{REFERENCES}

[1] Y. Nakamura, Y. A. Pashkin, and J. S. Tsai, "Coherent control of macroscopic quantum states in a single-Cooper-pair box," Nature, vol. 398, pp. 789-788, Apr. 1999.

[2] J. M. Martinis, S. Nam, and J. Aumentado, "Rabi oscillation in a large Josephson-junction qubit," Phys. Rev. Lett., vol. 89, p. 117901, Sep. 2002.

[3] I. Chiorescu, Y. Nakamura, C. J. P. M. Harmans, and J. E. Mooij, "Coherent quantum dynamics of a superconducting flux qubit," Science, vol. 299, pp. 1869-1872, Mar. 2003

[4] K. K. Likharev and V. K. Semenov, "RSFQ logic/memory family: A new Josephson-junction technology for sub-terahertz-clock-frequency digital systems," IEEE Trans. Appl. Supercond., vol. 1, pp. 3-28, Mar. 1991.

[5] S. V. Polonsky, V. K. Semenov, and D. F. Schneider, "Transmission of single-flux-quantum pulses along superconducting microstrip lines," IEEE Trans. Appl. Supercond., vol. 3, pp. 2598-2600, Mar. 1993.

[6] Y. Hashimoto, S. Yorozu, Y. Kameda, and V. K. Semenov, "A design approach to passive interconnects for single flux quantum logic circuits," IEEE Trans. Appl. Supercond., vol. 13, pp. 535-538, June 2003.

[7] WRspice is available from [Online]. Available: http://www.wrcad. $\mathrm{com} /$ wrspice.html

[8] M. Jeffery, P. Xie, S. Whiteley, and T. Van Duzer, "Monte-Carlo and thermal noise analysis of ultra-high-speed high temperature superconductor digital circuits," IEEE Trans. Appl. Supercond., vol. 9, pp. 4095-4098, June 1999

[9] S. Nagasawa, Y. Hashimoto, H. Numata, and S. Tahara, "A 380 ps. 9.5 $\mathrm{mW}$ Josephson 4-kbit RAM operated at a high bit yield," IEEE Trans. Appl. Supercond., vol. 5, pp. 2447-2452, June 1995.

[10] S. Intiso, J. Pekola, A. Savin, Y. Deviatov, and A. KidiyarovaShevchenko, "RSFQ circuits for low noise $\mathrm{mK}$ applications," Supercond. Science and Tech., vol. 19, pp. S335-S339, May 2006.

[11] N. Yoshikawa and Y. Kato, "Reduction of power consumption of RSFQ circuits by inductance-load biasing," Supercond. Sci. Technol., vol. 12, pp. 918-920, Nov. 1999. 